\title{
Zur Theorie der historischen Konstitution des bürgerlichen Staates Heide Gerstenberger
}

\section{Wider die staatstheoretischen Vorurteile}

Zur Entwicklung einer historisch-materialistischen Staatstheorie bedarf es der Einheit von logischem und historischem Forschungsprozeß, und lediglich der Arbeitsteilung wegen können die beiden Aspekte vorübergehend schwerpunktmäßig gesondert vorangetrieben werden. Die im folgenden dargestellte historische Konstitution des bürgerlichen Staates (1) ist also nicht mißzuverstehen als eine theoretische Erklärung des bürgerlichen Staates. Wohl aber ist sie durchaus solchen systematischen Theorieansätzen kritisch gegenübergestellt, welche den bürgerlichen Staat allein aus den Strukturen des entfalteten Kapitalverhältnisses meinen ableiten zu düren. Denn der bürgerliche Staat ist nicht nur jener politische Überbau, welchen die bürgerliche Gesellschaft notwendigerweise aus sich hervorbringt, sondern er bildet in vieler Hinsicht zugleich deren Voraussetzung.

Diesen konkreten Zusammenhang zwischen der Entwicklung der bürgerlicher Gesellschaft und ihres Staates bekommt man in den Griff, wenn man sich löst von der Bestimmung des bürgerlichen Staates durch seine Form, also seiner durch Rechtsstaatlichkeit gekennzeichneten und auf Parlamentarismus hin tendierenden Verfassung.

In allen europäischen Nationalstaaten vollzieht sich die reale Entwicklung von Staatsfunktionen für die bürgerliche Gesellschaft bereits in der merkantilen (2) Phase, und die Durchsetzung der formalen Konstitution des bürgerlichen Staates folgt dieser Entwicklung historisch nach. Nur für die USA fallen beide Konstitutionsprozesse zusammen, und aus dieser historischen Gleichzeitigkeit erklärt sich die dortige deutlich ökonomische Fundierung von Verfassungstheorie, die in den europäischen

1) Wenn hier von der Konstitution des bürgerlichen Staates die Rede ist, so bezieht sich dies auf den in der Zeit vom 15. bis 19., vor allem aber in den beiden mittleren Jahrhunderten sich vollziehenden Entwicklungsprozeß in Europa und Nordamerika. Außer Betracht bleiben dabei vor allem zwei Formen bürgerlicher Staatsorganisation: die Stadtstaaten I taliens im 14. und 15. Jahrhundert sowie die nachkolonialen Staatsgründungen des ausgehenden 19. und unseres Jahrhunderts. Letztere erfordern einen eigenen historischen Erklärungsansatz, weil es sich um nationale Übernahmen bereits etablierter bürgerlicher Herrschaftsapparate handelt; erstere sind auszuklammern, weil ihnen gerade die Auswe: tung zum einheitlichen Flächenstaat versagt blieb, die kennzeichnend ist für den modernen Nationalstaat. Eben jene geographische Verlagerung der WeIthandelswege, welche vom ausgehenden 15. Jahrhundert an die Entstehung der modernen Nationalstaaten förderte, bedeutete gleichzeitig den ökonomischen und politischen Niedergang der vordem mächtigen Stadtrepubliken in Italien. Was hier behandelt wird ist demzufolge die Konstitution weitgehend autonomer bürgerlicher Staaten.

2) ,Merkantil' steht im gesamten Aufsatz für eine vorwiegend durch räuberische Ausbeutung und Handel gekennzeichnete Akkumulationsform und bezeichnet zugleich die auf derartige Akkumulationsweisen gerichtete staatliche Politik. Der im Deutschen gebräuchlichere Ausdruck ,merkantilistisch" wird nicht benutzt, weil er durch die neuere wirtschaftspolitische Diskussion zu einem rein formalen verkommen ist. 
Emanzipationstheorien des Bürgertums im Kampf um Rechtsstaat und Parlamentarismus zunehmend zurïcktritt (3).

Die gängige Ineinssetzung von formaler und materieller Konstitution des bürgerlichen Staates wirkt sich in der bisherigen staatstheoretischen Diskussion vor allem in zweierlei Hinsicht aus. Zum einen führt sie dazu, daß die Legitimationsfunktion des bürgerlichen Staates ins Zentrum der theoretischen Diskussion gestellt wird. Denn in der spezifischen Form des bürgerlichen Staates kommt - das ist der richtige Inhalt der klassischen bürgerlichen Theorien von der Begnündung des Staates - dessen Legitimationsfunktion zum Ausdruck. Zum anderen verdunkelt dieses theoretische Vorurteil die materielle Relevanz unterschiedlicher konstitutioneller Formen des Staates.

Ebensowenig wie davon ausgegangen werden darf, daß die materielle Konstitution des bürgerlichen Staates erst im Zusammenhang mit der verfassungsmäßigen sich durchsetzen läßt, kann angenommen werden, daß eine konsequente bürgerliche, also parlamentarische Verfassung, zugleich die bestmöglichste Erfüllung staatlicher Funktionen für die bürgerliche Produktionsweise bedeute.

Derartige Vorurteile hinsichtlich der formalen verfassungsrechtlichen Organisation haben die Forschung aber bis in die jüngste Zeit hinein bestimmt. Sie lassen sich historisch zurïckführen sowohl auf die Schriften frühbürgerlicher Theoretiker als auch auf bestimmte (m. E. vor allem strategische) Außerungen von Marx und Engels (4). Obwohl deren Åußerungen zunächst nicht inehr besagten, als daß der Parlamentarismus die vollständigste Entsprechung darstellt der Theorie der bürgerlichen Gesellschaft, und diejenige politische Form ist, in welcher sich diese Gesellschaft am konsequentesten entfalten kann, sind sie - ungepruft - zunehmend auch dahin interpretiert worden, daß der Parlamentarismus dem Wachstum und dem Bestand der kapitalistischen Produktionsweise besonders kongruent sei. Es gibt genügend Arbeiten, in welchen die monarchische Verfassung Frankreichs im 18. und die autokratische Verfassung Preußen-Deutschlands im 19. Jahrhundert von vornherein als Hemmnisse für die Durchsetzung kapitalistischer Interessen gewertet werden.

Der Zusammenhang zwischen Basis und Überbau ist aber nicht so starr und eindimensional, als daß eine politische Form, welche der Herrschaftsorganisation der bürgerlichen Gesellschaft und deren Ideologie am voliständigsten entspricht, damit auch schon als die bestmögliche Erfüllung von politisch-ökonomischen Bedingungen der kapitalistischen Produktionsweise vorwegdefiniert wäre.

3) Obwohl die USA Nachfolgestaat eines Kolonialgebietes sind, werden sie hier generell im Zusammenhang der ,autonomen' bürgerlichen Staatskonstitution behandelt. Dies erklärt sich aus dem wesentlichen Unterschied britischer merkantiler Kolonialherrschaft im 17. Jahrhundert und auch noch im 18. Jahrhundert zu späteren (und gleichzeitigen iberischen) Formen kolonialer Ausbeutung. Die Besonderheiten des amerikanischen Konstitutionsprozesses werden ausführlich behandelt in: Heide Gerstenberger, Zur politischen Ökonomie der bürgerlichen Gesellschaft. Die Bedingungen ihrer historischen Konstitution in den USA. Frankfurt a. M., Oktober 1973

4) Diese Feststellung bezieht sich vor allem auf die Schrift: Der Achtzehnte Brumaire des Louis Bonaparte, MEW Bd. 8 
Eine nicht an der Form, sondern an der Funktion des bürgerlichen Staates festgemachte Theorie durchbricht derartige in der Forschung zumeist unreflektiert auftretende Ausgangshypothesen und bemüht sich um die Analyse des jeweils konkreten Zusammenhanges von Staatsfunktionen und ökonomischer Entwicklung Für Analysen dieser Art soll im folgenden ein systematischer Erklärungsansatz vorgetragen werden. Dabei werde ich nicht nur die wichtigsten Komponenten des realen Konstitutionsprozesses darzustellen suchen, sondern zugleich eine Erklärungshypothese entwickeln für das Auseinanderfallen von materieller und formaler Konstitution des bürgerlichen Staates.

Eine Vorbemerkung ist noch notwendig: hier wird zwar keine systematische Begründung der Staatstheorie vorgelegt, wohl aber ein systematisierender Erklärungsansatz für Entwicklungsprozesse, welche im einzelnen durchaus unterschiedlich verliefen. Wir bewegen uns also auf einer Abstraktionsebene, die von den konkreten Kämpfen absieht, von welchen die Durchsetzung der bürgerlichen Gesellschaft jeweils begleitet war. Innerhalb des hier vorgelegten allgemeinen Erklärungsansatzes bestimmen sich die besonderen Bedingungen einzelner nationaler Entwicklungen vor allem durch die folgenden, miteinander zusammenhängenden Komponenten: die reale und potentielle Stellung am Weltmarkt (dies resultiert aus der Dominanz der merkantilen Akkumulation in jener Zeit), aus den konkreten Kämpfen zwischen den Klassen und aus dem bereits erreichten Differenzierungsgrad der Kapitalfraktionen.

\section{Die Konstitu tion des bürgerlichen Staates als merkantile Außenvertretung}

Die frühen Theoretiker der bürgerlichen Produktionsweise haben ebenso wie deren schärfste Kritiker darauf hingewiesen, daß historisch die Durchsetzung des Kapitalismus vor allem zwei konstitutive Voraussetzungen hat: die private Akkumulation von Geld und die Herauslösung der Arbeitskraft aus den feudal-agrarischen Produktionsverhältnissen (5). Unter dem Begriff der ursprünglichen Akkumulation sind diese Bedingungen der bürgerlichen Produktionsweise inzwischen vielfach analysiert worden. Während jedoch bislang vor allem die gesellschaftliche Form der ursprünglichen Akkumulation den Schwerpunkt des wissenschaftlichen Interesses bildete, haben wir uns hier zu konzentrieren auf die spezifischen politischen Mittel, auf die legislativen, administrativen und militärischen Formen staatlicher Politik durch die

5) Dies ist eine sehr allgemeine Formulierung. Sie besagt nicht, dals akkumulierende Handelskapitalisten als solche Initiatoren der kapitalistischen Produktionsweise (also Unternehmer) wurden, sondern daß private Akkumulation eine der Voraussetzungen bildete zur erfolgreichen Durchsetzung der kapitalistischen Produktion. Letztere konnte aber auch durch die Weiterentwicklung von Handwerksbetrieben und durch die Ausnutzung des Kapitalmarktes erfolgen (s. u. Behandlung der Banken als Entwicklungsinstrumente). Neben diesen Formen lassen sich teilweise aber auch personelle Kontinuitätslinien in der Entwicklung von Handels- zu Verlags- und schließlich zu Industrieunternehmungen nachweisen. VgL hierzu z. B. die Angaben in der sehr guten Fallstudie von Friedrich Lohmann, Die staatliche Regelung der englischen Wollindustrie vom XV. bis zum XVIII. Jh., Leipzig 1900, S. 7 
hindurch die ursprïngliche Akkumulation sich vollzog. Anders formuliert: die Frage nach der Entstehung staatlicher Funktionen für die bürgerliche Organisation der Produktion erfordert eine Auseinandersetzung mit dem realen historischen Inhalt dessen, was heute zumeist völlig unhistorisch als merkantilistische Politik bezeichnet wird (6). Denn in die Phase des Merkantilismus fällt die Entwicklung der ursprünglichen Akkumulation, die fortan ein konstitutives Merkmal der kapitalistischen Produktionsweise werden sollte.

Der Merkantilismus ist jene historische Epoche, in welcher sich die Warenproduktion im Zusammenhang mit der Entwicklung der äußeren Märkte (7) durchsetzte. Die wichtigste Quelle der privaten Akkumulation an Geld bzw. Kapital bildete in dieser Zeit die staatlich unterstuitzte oder organisierte merkantile Eroberung (8). Diese Behauptung läßt sich nicht nur systematisch ableiten, sie ist auch vielfach statistisch belegt worden. Und zwar sowohl von den großen Verfechtern merkantiler Handelspolitik, wie etwa Davenant, Postlethwayt oder Colbert (9), als auch von Historikern der jüngsten Zeit, wie Elisabeth Schumpeter, Phyllis Deane, Cole oder Davis (10). Während die Analyse des Handelsvolumens in Relation zum Gesamtwachstum des Nationaleinkommens bei den modernen Arbeiten statistisch ungleich zuverlässiger ist als bei den Autoren des 17. und 18. Jahrhunderts, wird bei den letzteren noch deutlich, daß vieles, was heute unter dem neutralen Begriff des Handels erscheint, aufzuschlüsseln ist zum einen als Handel mit sog. schwarzem Elfenbein, mit Sklaven also, zum anderen nur bezeichnet werden kann als gewaltsame Aneignung. Exemplarisch ist zu erinnem an die Gewinnungsmethoden für Edelmetalle, wie sie in den spanischen Besitzungen Südamerikas zur Anwendung kamen.

Hier ist jedoch nicht der Ort, die Entwicklung der äußeren Märkte im einzelnen darzustellen; vielmehr ist hinzuweisen auf deren doppelte Funktion. Sie waren zum einen Lieferanten von Rohstoffen aller Art, von Edelmetallen, Spezereien und Sklaven: damit bildeten sie eine Quelle für Reichtum, die nur geringe Gegenleistung erforderte (11). Zum anderen entwickelten sich die äußeren Märkte jedoch zunehmend als Absatzgebiete von Manufakturwaren und bildeten damit eine Vorausset-

6) Als Einführung in den Stand der Diskussion ist geeignet: D. C. Coleman, Hrsg., Revisions in Mercantilism. London 1969.

7) Mit dem Begriff der äußeren Märkte soll vor allem der Tatsache Rechnung getragen werden, daß im 17. und 18. Jahrhundert noch keineswegs alle Handelsgebiete tatsächlicher Kolonialbesitz waren.

8) Vgl. auch Wolfram Fischer, Ökonomische und soziologische Aspckte der frühen Industrialisierung, in: Wirtschafts- und Sozialgeschichtliche Probleme der frühen Industrialisierung. Berlin 1968, S. 9

9) Charles Davenant, An Essay upon the Probable Methods of Making a People Gainers in the Balance of Trade. 2. Ed., 1700, o. O.; Daniel Defoe, A Plan of the English Commerce, London 1728; Malachy Postlethwayt, The Universal Dictionary of Trade and Commerce. London, 2 Bde., 1766; Pierre Clément. Ed., Lettres, instructions et mémoires de Colbert. 410 vols., Paris 1859 ff.; Thomas Mun, England's Treasure by Foreign Trade, 1665

10) Literaturangaben s. u. Anmerkung 12

11) Die vorkapitalistische Akkumulation resultierte überwiegend aus merkantilen Situationsgewinnèn im Außenhandel. Daß dieser Zusammenhang in der Theorie der Handelsbilanz, wie sie die Merkantilisten des ausgehenden 17. und des 18. Jahrhunderts vertraten, weit- 
zung für den erfolgreichen Kampf der Bürger gegen jene feudalen Produktionsbeschränkungen, die in den Zunftordnungen versteinert waren (12).

In unserem Zusammenhang ist die Eroberung von äußeren Märkten nicht nur bedeutsam als die hervorragendste Quelle des Reichtums bzw. des Handelskapitals unter spätfeudalen und frühkapitalistischen Bedingungen, sondern als die zentrale ökonomische Ursache der Konstitution des bürgerlichen Staates. Denn der Kampf um die äußeren Märkte ließ sich nicht durchfuihren mit privaten noch mit merkantilen (also handelspolitischen) Mitteln allein. Er erforderte Flotte und Heer, ein geordnetes nationales Geldsystem, ein Kreditsystem, sowie die Übernahme staatlicher Garantien für private Vertragsabschlüsse mit quasi-öffentlichem Charakter in den überseeischen Gebieten.

Voraussetzung zur Behauptung im internationalen Konkurrenzkampf um die äußeren Märkte war sowohl ein bestimmtes Quantum an privat akkumuliertem Handelskapital als auch eine relativ finanzstarke oder doch kreditwürdige Zentralgewalt. Und diese fiskalischen Voraussetzungen erklären vieles, was heute als Prinzipienstreit der Merkantilisten über Protektionismus oder Freihandel mißinterpretiert wird. In einer seiner verdienstvollen Arbeiten hat Ralph Davis nachgewiesen (13), daß die Entwicklung des Protektionismus in England in den Jahren 1689-1786 in ihren einzelnen Maßnahmen jeweils direkt zurückzuführen ist auf unmittelbare Finanzbedürfnisse der Regierung. Und diese Finanzbedürfnisse resultierten ihrerseits aus der Notwendigkeit, teure Kolonial- und Handelskriege zu führen. In diesem Zusammenhang ist daran zu erinnem, daß die großen europäischen Kriege des 17. und 18. Jahrhunderts im wesentlichen ebenfalls zu begreifen sind als Handels-

gehend richtig erfaßt wurde, läßt sich hier nicht austühren. Die heute vielfach gegen diese Auffassung vorgebrachte These vom automatischen Zahlungsbilanzausgleich läßt unberücksichtigt, daß damals Edelmetalle dazu tendierten, durch Schmuckverwertung und Schatzbildung der Zirkulation entzogen zu werden. Vgl. hierzu: Rudolf Blitz, Mercantilist Policies and the Patterns of World Trade 1500-1750. The Journal of Economic History. Bd. 27, 1967, S. 39-56

12) Absatzgebiet für (unterschiedliche Sorten von) Manufakturwaren waren sowohl die noch nicht in Besitz genommenen Handelsgebiete, wie etwa Indien, weite Teile Afrikas, als auch die vorwiegend monokulturell produzierenden (Westindische Inseln), und die vorwiegend Edelmetalle liefernden Kolonien (portugiesische und spanische Besitzungen in Südamerika). Für Großbritannien wurden als Abnehmer zunehmend wichtiger die nordamerikanischen Kolonien. Phyllis Deane und W. A. Cole haben für Großbritannien herausgestellt, daß Manufakturexporte bereits zu Beginn des 18. Jahrhunderts etwa $85 \%$ des britischen Gesamtexports ausmachten. Da dieser Exportanteil einem Drittel der gesarten Manufakturproduktion entsprach, messen Deane und Cole dem Manufakturexport eine besondere Bedeutung für die Entwicklung der Manufakturproduktion in Grołsbritannien zu (vgl. Phyllis Deane, W. A. Cole, British Economic Growth 1688-1959, 2 Ed., Cambridge 1967, S. 41). Diese Einschätzung stimmt mit der meinen überein; in seiner Analyse der von E. Schumpeter vorgelegten Daten schreibt Ashton, daß als herausragende Entwicklung des britischen Handels im 18. Jahrhundert der relative Bedeutungsschwund traditioneller Märkte und die Bedeutungszunahme neuer Import- und Exportgebiete festzuhalten sei. Vorwort von T.S. Ashton zu Elizabeth Boody Schumpeter, English Overseas Trade Statistics 1697-1808. Oxford 1960, S. 10

13) Ralph Davis, The Rise of Protection in England, 1689-1786, in: Economic History Review, Nr. 19, 1966, S. 306-317 
kriege um die Dominanz auf den äußeren Märkten. Wohl wurde auch gekämpft um die Vorherrschaft am europäischen Markt, aber wenn sich etwa in den spanischen Erbfolgekriegen England mit Portugal gegen Frankreich verbündete, so bedeutete dies nicht nur, daß statt der französischen jetzt die portugiesischen Weinimporte nach Großbritannien gefördert wurden und englische statt der französischen Wollmanufakturwaren in Portugal dominierten. Es bedeutete vor allem, daß Großbritannien den Löwenanteil des direkten und des über Portugal laufenden indirekten Exportes nach Brasilien für sich durchgesetzt hatte. Welche ungeheure ökonomische Bedeutung diese hier nur exemplarisch herangezogene Handelsvorherrschaft während der Hochkonjunktur der brasilianischen Gold- und Diamantenproduktion im 18. Jahrhundert hatte, ist in einer 1971 erschienen Arbeit von Harold Fisher dargestellt worden (14).

Was hier und im übrigen auch in den Schriften englischer Merkantilisten als nationale Interessen behandelt wird, ist politisch zu differenzieren. Zwar waren in allen großen Außenhandelsnationen die Krone, das Bürgertum und der Adel an den merkantilen Unternehmungen beteiligt, doch die jeweilige Relation dieser Beteiligung unterschied sich beträchtlich von Land zu Land. Waren die portugiesisch-spanischen Kolonisationen Unternehmungen der Krone, die dem Adel Sinekuren in der Kolonialverwaltung verschafften und Vertreter des Bürgertums hauptsächlich am Warentransport beteiligten, so erwies sich als auf Dauer weitaus erfolgsträchtiger das vor allem in England und Frankreich entwickelte Verfahren der Privilegierung selbständig agierender vorwiegend bürgerlicher Handelskompanien (15). In diesen Ländern kam das sog. .nationale" Interesse zustande durch die Kongruenz von Interessen bürgerlicher Handelskompanien an einer militärisch und finanziell starken Z \&ntralmacht mit Interessen der Krone, welche sich Unabhängigkeit zu verschaffen suchte von der Notwendigkeit politischer und finanzieller Unterstützung durch die Feudalaristokratie und ihrer Körperschaften (16). Das Interesse an der Entwicklung der äußeren Märkte trug in diesen Ländern dazu bei, die Umwälzung feudaler Strukturen in national-bürgerliche zu begünstigen, wobei zu berücksichtigen ist, daß der hiermit verbundene relevante Zentralisierungsprozeß sich nicht nur gegen die Feudalaristokratie, sondern auch gegen die Städte, die frühen Hochburgen bürgerlicher Unabhängigkeit, zu richten pflegte (17).

14) H.E.S. Fisher, The Portugal Trade. A Study of Anglo-Portuguese Commerce 1700-1770. London 1971

15) Eine hervorragende Untersuchung der unterschiedlichen Organisationsformen (und klassenspezifischen Beteiligungen an) der merkantil-kolonialen Ausbeutung findet sich bei: E. L. J. Coomaert, European Economic Institutions and the New World: the Chartered Companies, in: The Cambridge Economic History of Eurcpe. Bd. IV. Cambridge 1967, S. $223-273$

16) Besonders deutlich ist dieser Zusammenhang für Frankreich. Vgl. hierzu: Lionel Rothkrug, Opposition to Louis XIV, The Political and Social Origins of the French Enlightenment. Princeton, New Jersey 1965, S. 86 ff.: siehe hierzu auch: Eugene L. Asher, The Resistance to the Maritime Classes. The Resistance of Feudalism in the France of Colbert. Berkeley - Los Angeles 1960

17) Der Kampf Colberts und seiner Nachfolger mit den Städten ist bekannt. Er war mit absolutistischen Mitteln erfolgreicher als die republikanischen Instanzen der Generalstaa- 
Die Validität der hier vorgetragenen Hypothese von der Konstituierung des bürgerlichen Staates als merkantiler Außenvertretung beweist sich durch ihren Erklärungswert: Sobald wir zu ermitteln suchen, welchen Gebieten in Europa durch geographisch-klimatische Bedingungen und/oder durch bestimmte Ausprägungen ihrer Produktionsschwerpunkte die Eroberung äußerer (präziser: außereuropäischer) Märkte erschwert war, so stellen wir fest, daß sie sich decken mit denjenigen Gebieten, in welchen die Entwicklung zum modemen Nationalstaat retardierte. Für den Fortgang der Entwicklung können wir dariberhinaus feststellen, daß jene spezifischen Formen autokratischer Staatlichkeit, welche vor allem für Preußen-Deutschland üblicherweise zurückgeführt werden auf eine nationale Verspätung, ihren Ursprung haben in der ökonomischen Notwendigkeit, privat nicht zu erzielende Resultate der ursprünglichen Akkumulation subsidiär durch staatliche Macht herzustellen (18). Derartige Subsidiarität ging, wie Schmoller gezeigt hat, so weit, daß in Preußen im 18. Jahrhundert von Staats wegen der Versuch unternommen wurde, Handels- und Finanzgesellschaften zu gründen, wie sie andernorts als Zusammenschlüsse von Handelsbürgern zur Entstehung kamen (19).

Das auch in historisch-materialistischen Arbeiten noch gängige Phasenschema der Staatstätigkeit, welches davon ausgeht, daß während der Phase der Entfaltung des Kapitalismus sich die Akkumulationsbedingungen des Kapitals durch das Wirkep der Konkurrenz ohne Staatseingriffe herstellten, die Staatstätigkeit demnach auf die Erstellung von Rahmenbedingungen für die kapitalistische Produktionsweise beschränkt bleiben konnte, ist ein Reflex der (von Mitte des 18. Jahrhunderts bis gegen Ende des 19. Jahrhunderts andauernden) Vormachtstellung des britischen Kapitals auf dem Weltmarkt. Nur in England stellten sich aufgrund der Dominanz am Weltmarkt Bedingungen her, welche die Entfaltung der freien Konkurrenz und schließlich die Freihandelspolitik ohne Schaden für das nationale Kapital ermöglichten. Die nationalen Akkumulationsbedingungen des Kapitals sind von allem anfangan durch den Weltmarkt geprägt, und was die Staatstätigkeit anbelangt, so bildet die englische Entwicklung nicht so sehr den ,klassischen Fall ${ }^{\prime}$ als vielmehr den auf Weltmarktdominanz beruhenden besonderen.

Da es sich hier lediglich um die Begrüindung einer Hypothese, nicht jedoch um

ten. Daß in den Niederlanden in der Utrechter Union vom 25.1. 1579 den Städten zu viel Macht belassen wurde, hält Pringsheim für eine der Ursachen des ökonomischen Niederganges. Vgl. Otto Pringsheim, Beiträge zur wirtschaftlichen Entwicklungsgeschichte der Vereinigten Niederlande im 17. und 18. Jahthundert. Leipzig 1890, S. 1 ff. und 26.

18) Vg1. hierzu auch: Max Adler, Die Anfänge der merkantilistischen Gewerbepolitik in Österreich, in: Wiener Staatswissenschaftliche Studien Bd. 4, Wien-Leipzig 1903, S. 5 ff. Adler sieht die späte Entwicklung des Merkantilismus in Österreich.im Zusammenhang mit den lange fehlenden äußeren Märkten (Widerstandskraft des Osmanischen Reichs).

19) Gustav Schmoller, Die Einführung der französischen Regie durch Friedrich den Großen; Sitzungsberichte der königlichen Preußischen Akademie der Wissenschaften zu Berlin, Nr. IV, 1888, S. 70. Zur Bedeutung der ,Seehandlung“ (einer zunächst vor allem als staatlich privilegierte Handelsunternehmung, später zunehmend als Finanzierungsinstitut eingesetzten Gründung) vgl auch W. O. Henderson: The State and the Industrial Revolution in Prussia 1740-1870. Liverpool 1958, S. $120 \mathrm{ff}$. 
eine ins einzelne gehende Darstellung handeln kann, soll jetzt nicht näher eingegangen werden auf die besonderen Schwierigkeiten der Entwicklung in Nordeuropa (20), noch auf die Erklärung der holländischen und später vor allem der britischen Vorherrschaft auf dem Weltmarkt. Stattdessen soll auf der angeschnittenen allgemeinen Ebene die abstrakte Funktionsbestimmung der Staatsmacht für die merkantile Form der Akkumulation weitergeführt werden. Dabei lassen sich die zunächst zu behandelnden Staatsaufgaben, die Bereitstellung einer Streitmacht und die Beschaffung von Ressourcen begreifen als eine Konkretion der als konstitutiv bereits herausgestellten Außenfunktion.

Der Einsatz der Staatsmacht diente der Sicherung bereits errungener Handelsgebiete, dem Schutz der Handelsschiffe vor Piraterie, der Außenvertretungen des Handelskapitals vor feindlichen Überfällen. Daneben aber waren alle am Weltmarkt beteiligten Nationen bestrebt, den größten Anteil am Welthandel für sich zu erringen. Es wird später noch darauf zurückzukommen sein, daß in der Phase der Konstitution des bürgerlichen Staates die nationale Souveränität als prinzipiell unbegrenzt begriffen wurde. Die Theorie von der sog. Zuhöchstheit bezieht sich einerseits auf die Position des Monarchen in seinem Volk, sie bezieht sich andererseits auf die Außenvertretung der Nation als Ganzes. Und hier ist die Souveränitätstheorie deutlich verankert in den politisch-ökonomischen Strukturen des merkantilen Systems.

Die zeitgenössischen Theoretiker des Merkantilismus gingen davon aus, daß die Reichtümer dieser Erde begrenzt seien und unter vorindustriellen Produktionsbedingungen war diese Annahme keineswegs unrealistisch. So galt es also nicht nur, den größten Anteil an den mehr oder minder natürlichen Schätzen der Erde unmittelbar zu sichern, sondern zugleich daraus zu profitieren, daß man diese Güter möglichst günstig an andere Nationen weiterverkaufte. In diesem Zusammenhang sind ein Großteil der sog. Navigations- und der Kolonialgesetze des 17. und 18. Jahrhunderts zu sehen, welche jeweils die Mutterländer als Zwischenhandelsstationen vorschrieben oder zu etablieren suchten. Je verschiedener und zahlreicher die Güter waren, über deren Welthandelsangebot ein Land bestimmen konnte, und je größer der Bereich der potentiellen Abnehmer, um so größer war die Möglichkeit, Situations- und Monopolgewinne aus dem Handel zu schlagen. Mit zunehmender Kapitalakkumulation entwickelte sich die handelskapitalistische und koloniale Herrschaft darüberhinaus immer mehr als Garant für den Absatz heimischer Manufakturwaren. In jedem Fall kann für das gesamte 17. und auch noch für das 18. Jahrhun. dert festgestellt werden, daß friedliche Beziehungen nur dann angestrebt wurden, wenn militärische Unterstützung merkantiler Expansion aussichtslos erschieĩ. Selbst in sog. Friedenszeiten allerdings waren Seeräuberei und Schmuggel übliche und von ehrenwerten Bürgern oft in großem Ausmaße betriebene Unternehmungen (21).

Der aktuelle oder doch zumindest ständig potentiell notwendige Einsatz von

20) Sie stehen unter anderem im Zusammenhang mit der merkantılen Ausbeutung durch die Hanse, welche bis zum ausgehenden 15. Jahrhundert den Ost-West-Handel des nördlichen Europa beherrschte.

21) Vgl. hierzu z. B.: Georg Schanz, Englische Handelspolitik gegen Ende des Mittelalters. 2 Bde., Leipzig 1881, Bd. 1, S. 253; Friedrich Lohmann, Die staatliche Regelung der englischen Wollindustrie, a. a. O., S. 75; Otto Pringsheim, Beiträge zur wirtschaftlichen 
Militär- und Seestreitkräften war nur zu gewährleisten, wenn die machtpolitischen Aktionen der Zentralregierung Unabhängigkeit erreichten von den Feudalherren, welche nur ungern die ihnen untertanen Bauern zum nationalen Kriegsdienst verpflichteten (22). Das stehende Heer bzw. die nationale Flotte, welche allein solche Unabhängigkeit und darüberhinaus die Souveränität der Nation zu garantieren vermochten, erforderten aber Finanzmittel, welche die Möglichkeiten der königlichen Privatschatullen auf Dauer zu sprengen drohten.

So ist die Entwicklung der merkantilen Außenfunktion des Staates allenthalben begleitet von grundlegender Veränderung des Systems der Ressourcenbeschaffung. Dabei wurden nicht nur neue Geldquellen in stärkerem Umfang dem Staatsbudget erschlossen, vor allem änderte sich die Technik der Ressourcenbeschaffung, und die letztere Veränderung bedingte ihrerseits zunehmend die Entwicklung eines bürokratischen Apparates im Dienste der Zentralregierung (23).

Die Entstehung des modernen Steuersystems ist in mehr als einer Hinsicht zentral fuir die Konstitution des bürgerlichen Staates. Denn sie bildete nicht nur die Voraussetzung für die erfolgreiche Erfillung der merkantilen Außenfunktion, war nicht nur der bedeutsamste Anlaß für die Entwicklung der modernen staatlichen Administration, sondern sie hat darüberhinaus unmittelbar akzellerierend die ökonomische Entwicklung beeinflußt. Dies gilt bereits für den ersten Schritt der Veränderung des herkömmlichen Steuersystems; denn noch während der Fortgeltung feudaler Abgabenordnungen hat deren Umwandlung in Geldsteuern und die Erhöhung der letzteren dazu beigetragen, daß der Grund und Boden vieler vorher faktisch unabhängig produzierender Bauern an die Grundherren zurückfiel bzw. von bürgerlichen Großgrundbesitzern aufgekauft wurde; die verarmte Landbevölkerung stand entweder über eine Teilbeschäftigung im Verlag oder aber durch Abwanderung in die Städte den Manufakturbetrieben als Arbeitsangebot zur Verfugung (24). Die Veränderung des Steuersystems hat die Proletarisierung eines großen Teils der Landbevölkerung von Land zu Land in unterschiedlichem Ausmaß beeinflußt. Überall jedoch ist durch diesen Wandel der Ressourcenbeschaffung zugleich die Warenproduktion und die Ausbreitung des Manufaktursystems gefördert worden.

Entwicklungsgeschichte der Vereinigten Niederlande im 17. und 18. Jahrhundert, a. a. O., S. 3. Der Schmuggel ist zudem Thema fast aller größeren merkantilistischen Programmschriften.

22) Beispielhaft deutlich wird diese Entwicklung in England während der Regentschaft Heinrichs VIII., in Frankreich zur Zeit Philipps des Schönen, in Preußen unter dem Großen Kurfuirsten.

23) Hier wird also anders als bei Max Weber nicht so sehr die Entwicklung des modernen Staates aus der Bürokratisierung erklärt, als vielmehr die Entwicklung der Bürokratie aus bestimmten Staatsfunktionen. Letzterer Zusammenhang ist bei Max Weber implizit enthalten, wird theoretisch aber nicht erfaßt. Max Weber, Soziologie -- Weltgeschichtliche Analysen-Politik (Teilsammlung). Stuttgart 1968, 4. Aufl, vor allem S. $57 \mathrm{f}$, und $153 \mathrm{f}$. (Die drei reinen Typen der legitimen Herrschaft); ebenfalls: Max Weber, Wirtschaft und Gesellschaft. Abschnitt: Wesen, Voraussetzungen und Entfaltung der bürokratischen Herrschaft. 5. Aufl. (Studienausgabe), Tübingen 1972, S. $551 \mathrm{ff}$.

24) Vgl. hierzu: Alexis de Tocqueville, L'Ancien Régime et la Révolution. (Neudruck) Paris o. J. 
Die Herausbildung des modernen Steuersystems kann hier nicht ausführlich behandelt werden (25). Sie läßt sich in großen Zügen kennzeichnen als eine Entwicklung, welche ausgeht von den Bemühungen, die früheren Sondersteuern und Requirierungen zu überwinden durch eine möglichst breite und direkte Besteuemung (26). Da letztere sich politisch nur sehr schwer durchsetzen ließ, gingen die Regierungen vorzugsweise den Umweg der indirekten Steuer, der Akzise (27).

Auch diese wurde freilich nicht ohne Kampf durchgesetzt (in Preußen z. B. gelang die Durchsetzung der Akzise nur für die Städte; die Landbevölkerung (d. h. vor allem die Grundbesitzer und die von ihnen abhängigen Schichten) war bis 1873 von der Akzisenregelung - vor allem der Mahl- und Schlachtsteuer - befreit (28). Insbesondere in Holland, England, den deutschen Gebieten und Österreich schlug der Akzisenstreit heftige politische Wellen. Er bewirkte zugleich, daß die Theoretiker der nationalen Ökonomie sich bessere Klarheit verschafften über die ökonomische Wirkung von Steuern (29). In diesem Zusammenhang stand auch die Auseinandersetzung um die Zölle, die neben Akzisen und Luxussteuern die dritte wichtige indirekte Einnahmequelle der Zentralregierung bildeten. Zölle waren im Mittelalter und in der Frühzeit des Merkantilismus vorwiegend, wenn auch nicht ausschließlich, zu fiskalischen Zwecken erhoben worden. Erst mit Ausweitung des Warenverkehrs und der Entwicklung anderer Einnahmequellen wurden Zölle zunehmend betrachtet als Mittel der Gewerbepolitik (30), zur Steuerung des Vorrats an Rohstoffen und zur Sicherung von Absatzmärkten für die heimische Industrie (31). Der große theoretische und praktische Fortschritt, der in der Entwicklung

Vgl. zum Gesamtkomplex vor allem: Gabriel Ardant, Théoric Sociologique de l'Impôt 2 Bde, Paris 1965

26) In diesem Zusammenhang ist vor allem zu erinnern an die Bemühungen Turgots und seiner Nachfolger in Frankreich. In England sind ähnliche Ziele von Cromwell verfolgt worden.

27) Vgl. hierzu: Gabriel Ardant. Théoric Sociologiquue de IInpôt. a. a. O., S. 621; Gustav Schmoller, Skizze einer Finanzgeschichte von Frankreich, Österreich, England und Preußen 1500-1900. Leipzig 1909: B. E. V. Sabine, A History of Income Taxe. London 1966. S. 18

28) Vgl. hierzu: O. Schwarz, J. Strutz, Der Stadtshaushait und die Finanzen Preußens. Berlin 1902. Achtes Buch. 1. Abschnitt. Erwin von Beckerath: Die preußsische Klassensteuer und die Geschichte ihrer Reform bis 1851 . Munchen-Leipzig 1912.S. 3 ff.

29) In Ergänzung zu den bereits angeführten Arbeiten sei hier verwiesen auf: Johann Heinrich Gottlob Justi: Ausfïhrliche Abhandlung von den Steuern und Abgaben. Königsberg-Leipzig 1762: Victor Riquetti de Mirabeau. Théoric de l"Inpôt. o. O. 1760

30) Trotzdem wurden auch aus fiskalischen Gründen noch Zölle beibehalten, so u. a. das (früher bullionistisch bedingte) Verbot der Edelmetallausfuhr. Dieser Iimstand hat vielfach dazu geführt, dal3 in der Literatur auch den späten Merkantilisten noch unterstelt wird, sie hätten Reichtum mit Edelmetallbesitz identifiztert.

31) VgL hierzu besonders: Friedrich Lohmann, Die staatliche Regelung der englischen Wollindustrie, a. a. O., S. $65 \mathrm{ff}$. Lohmann zeigt auf, dafo vom 13. bis zum 15. Jahrhundert teilweise und absolute Wollausfuhrverbote lediglich aus fiskalischen Grüden erlassen wurden. Die Regierung konnte so durchsetzen. dał einerseits durch die hohen Zölle die Einnahmen stiegen, andererseits durch den Verkauf des Privilegiums der Wolausfuhr. Erst später seien derartige Maßnahmen als wirtschaftspolitische getroffen worden. 
vom bloßen Fiskalismus zur staatlichen Wirtschaftspolitik in der veränderten Behandlung der Zölle enthalten ist, prägt auch die Diskussionen um die übrigen Staatseinnahmen. So haben vor allem die französischen Steuerreformer wie Boisguillebert, Vauban und Turgot die Überzeugung vertreten, daß die finanzielle Sicherung der Krone um so erfolgreicher zu gewährleisten sei, je mehr diese auf die ihr zustehenden feudalen Abgaben verzichte und stattdessen die Entwicklung der Marktbeziehungen und den Erfolg der privaten Unternehmungen ihrer Bürger fördere (32).

In unterschiedlichen Ausprägungen sehen wir deshalb überall einen mehr oder minder deutlichen Übergang zu Nettoertrags-und Einkommenssteuern. Vielfach treten sie noch auf in der Form von (katastrierten) Aufwandssteuern (Fenster-, Tür-, Haarpuder-, Dienstbotensteuern z. B. (33)). Wo - wie 1789 in Großbritannien als Kriegssteuer - bereits die Einkommenssteuer eingeführt wurde, traf diese noch auf die Schwierigkeiten des staatlichen Konstitutionsprozesses. Denn diese allgemeine, direkte Besteuerung, mit der für sie charakteristischen Selbsteinschätzung der Bürger (im Unterschied zur früheren Taxierung von Amts wegen), erforderte zu ihrer Durchführung eine zentralisierte Verwaltung, welche nach gleichen Grundsätzen die Überwachungsfunktion und Richtlinienentwicklung ausfïllen konnte (34). Die historische Ungleichzeitigkeit der Durchsetzung der Einkommenssteuer, als der typisch bürgerlichen Steuerform, ist ein wichtiger analytischer Indikator für den jeweiligen nationalen Entwicklungsstand des bürgerlichen Staates (35). Auch hinsichtlich dieses Aspektes kommt die politisch-ökonomische Vorherrschaft (und damit der organisatorische Vorsprung) Großbritanniens voll zum Durchbruch.

Die staatlichen Finanzprobleme hatten Auswirkungen nicht nur bezüglich des Apparats der Steuer- und Zollbehörden, sie brachten vor allem nationale Kalkulationsprobleme mit sich. So ist die Entwicklung der nationalen Militärmächte begleitet von der Trennung der königlichen Privatschatulle und dem Staatsschatz, von der Kalkulation des Staatshaushalts sowie von ersten Berechnungen des Volkseinkommens (36). Mit gutem Grund hat sich die Theorie des merkantilen Systems deshalb

32) Vgl. hierzu: Charles Woolsey Cole, French Mercantilism, 1683-1700. New York 1965 (erstmals 1943), S. 230 ff.; ebenfalls: Gabriel Ardant, Théoric Sociologique de l'Impôt. a. a. O., S. $211 \mathrm{ff}$.

33) Zur Ertragssteuergesetzgebung der französischen Nationalversammlung vgl. Hermann Meyer, Die Einkommenssteuerprojekte in Frankreich bis 1887. Berlin 1905, S. 30 ff; vgl. hierzu auch: Franz Rompe, Die ideengeschichtliche Entwicklung des Einkommenssteuerproblems in Frankreich. Leipzig 1950; Zu den englischen Verbrauchssteuern des 18. Jahrhunderts vgl. B. E. V. Sabine, A History of Income Taxe, a. a. O., S. $19 \mathrm{f}$.

34) Vgl. hierzu: Herbert Arendt, Entwicklung und innerer Aufbau der englischen Einkommenssteuer. Jena 1931, S. 1-5; B. E. V. Sabine, A History of Income Taxe, a. a. O., S. 25

35) Theoretisch findet er sich reflektiert durch David Ricardo, On the Principles of Political Economy and Taxation, erstmals 1817 , insbes. Kap. 8-18

36) Sobald private Handelskompanien angegriffen wurden, weil sie zuviel Gold aus dem Lande beförderten, zuviele Manufakturwaren importierten und so einheimische Arbeitslosigkeit herautbeschworen etc., sahen sie sich bemüßigt, durch genaue nationale Gewinn- und Vetlustrechnungen ihre Unternehmungen $\mathrm{zu}$ rechtfertigen. In diesen Rechtfertigungsschriften, die hervorragendsten sind uns hinterlassen worden von den 
häufig als ,politische Arithmetik“" bezeichnet (37).

Solche nationale Buchfihirung bildete Voraussetzung und Hemmnis zugleich der zweiten finanzpolitischen Komponente im Konstitutionsprozeß des bürgerlichen Staates: der systematischen Politik der Staatsverschuldung als eines Mittels der nationalen ökonomischen Entwicklung (38).

Waren fruiher die Fürstenhâuuser Schuldner einzelner privater Bankiers gewesen, so überstieg das jetzt benötigte Kreditvolumen nicht nur die meisten privaten Möglichkeiten, die Kreditbeschaffung für die im Weltmaßstab betriebenen merkantilen und militärischen Unternehmungen mußte vor allem ihren eher zufälligen, von der Machtpolitik einzelner Handelshäuser abhängigen Charakter verlieren (39).

So bildete sich in den meisten europäischen Staaten (und später auch in den USA) im 18. Jahrhundert ein Finanzsystem heraus, in welchem die rechte Dosierung von öffentlichen Krediten, Staatsschulden und Umschuldungsregelungen mit gutem Grund für eine Kunst erachtet wurde. Und die Übernahme ökonomischer Funktionen für die bürgerliche Gesellschaft durch die Zentralgewalt läßt sich jeweils ablesen an den Kämpfen um die Errichtung einer Nationalbank (40). Denn als Instrument zur Regelung der Schulden des Staates bildet sie das Zentrum der Auseinandersetzungen um die Bedeutung des Staates für das Wachstum des nationalen Reichtums (41). Zwar überzieht Dickson den bestehenden Zusammenhang, wenn er meint, der Sturz der französischen Monarchie in der großen Revolution sei eine Folge des seit Colbert in Frankreich nicht mehr weiterentwickelten öffentlichen Finanzsystems gewesen, wohl aber ist ihm zuzustimmen, wenn er den Erfolg der britischen Kolonialkriege auch zurïckführt auf das in Großbritannien am weitesten fortgeschrittene System der Staatsverschuldung (sein hervorragendes Merkmal war die ausgewogene Relation von langfristiger und kurzfristiger Staatsverschuldung und die Entwicklung systematischer Umschuldungspläne (42)). Die öffentlich geförderte

Betürwortern der East-India-Company in Grolsbritannien, wurde die okonomische Theorie des Zeitalters am nachhaltigsten vorangetrieben. Für Literaturangaben vgl. Anm. 6

37) Vgl. z. B. William Petty, Several Essays in Political Arithmetik. 1699.

38) Zur Geschichte der Staatsschuld vgl. E. L. Hargreaves, The National Debt. (erstmals 1933) London 1966; eine besonders brilliante zeitgenössische Auseinandersetzung findet sich in Alexander Hamiltons, Report Relative to a Provision for the Support of Public Credit', January 9, 1790, abgedruckt in: The Reports of Alexander Hamilton, Hrsg. Jacob E. Cooke. New York 1964

39) Zwar nahm die Zahl der großen Anleihen bei privaten Handelshäusern ab, aber das gesamte 18. Jahrhundert hindurch fungierten die Handelskompanien als Kreditgeber für den Staat. Vgl. B. E. V. Sabine, A History of Income Tax., a. a. O., S. 620; siehe auch oben die Angaben zur Preußischen Seehandlung.

40) Paul Mantaux, The Industrial Revolution in the Eighteenth Centurury, (erstmals 1921) New York-Evanston 1962, S. 95 ff.

41) Vgl. hierzu die Auseinandersetzung mit Mc. Culloch in David Ricardo, Plan for the Establishment of a National Bank, 1824. Besonders heftig war die - als Verfassungsstreit gefihrte - Auseinandersetzung um die Nationalbank in den USA in den Jahren nach 1790.

42) P. G. M. Dickson, The Financial Revolution in England. A Study in the Development of Public Credit 1688-1756. New York 1967. Für die Entwicklung moderner Staatsfunktionen bietet diese Untersuchung eine hervorragende Fallstudie. 
Herausbildung eines Marktes für Wertpapiere war einerseits Voraussetzung für die erfolgreiche Akkumulation von Kapital im Kolonialhandel, gleichzeitig bewirkte sie jedoch die endgültige Zerstörung noch fortdauernder mittelalterlicher Zinsverborsvorstellungen - ein Umstand, der als ideologische Voraussetzung der kapitalistischen Entwicklung nicht zu unterschätzen ist.

Diente das System der Staatsverschuldung zunächst vorwiegend der Finanzierung nationaler Unternehmungen im merkantilen Interesse, so hat das in diesem Zusammenhang entstandene Kreditsystem zunehmend auch Bedeutung erhalten für die Finanzierung privater Unternehmungen. Diese Entwicklung stand ihrerseits in einem deutlichen Zusammenhang mit der merkantilen Machtstellung der Nation. Denn bereits seit dem 16. Jahrhundert läßt sich beobachten, daß Kapital jeweils in jene Länder transferiert wurde, in welchen die höchsten Profite zu erwarten waren. Mit diesem Hinweis auf die Kreditversorgung sei auf den sonst hier nicht ausgefuhrten Zusammenhang zwischen außenwirtschaftlicher Machtstellung und frühem Übergang zur kapitalistischen Produktionsweise aufmerksam gemacht.

Der sich herausbildende Weltmarkt fand seinen Ausdruck aber nicht nur in internationalen Kapitaltransfers. Vielmehr ist spätestens seit dem 16. Jahrhundert eine bewußte Politik der Auswertung fremder Erfahrungen und der Gewinnung ausländischen Sachverstands zu beobachten. So sind nicht nur italienische Fachleute nach Holland und Frankreich geholt worden, um dort Bankwesen und Börse bzw. die Seidenweberei zu entwickeln (43), auch ausländische Kaufleute und Bankiers gingen , bei den großen italienischen Handelshäusern in die Lehre (44). Sowohl auf privater als auch auf nationaler Ebene können wir zunehmend die bewußte Übernahme finanz- und wirtschaftspolitischer Instrumentarien bzw. Institutionen anderer Nationen beobachten (45). Und derartige Verbreitungsprozesse sind mit zu berücksichtigen, wenn es um die Analyse einzelner nationalgeschichtlicher Entwicklungen geht.

Dieser Hinweis soll verhindern, daß der hier vorgetragene Erklärungsansatz für die Konstitution des bürgerlichen Staates mißverstanden wird als ein historisches Ablaufmodell konkreter nationalstaatlicher Entwicklungsprozesse. Trotz der (im Vergleich zu historisch späteren Herausbildungen von Nationalstaaten) relativen

43) VgL hierzu: Germain Martain, La Grande Industrie sous le Règne de Louis XIV. Paris 1898 , S. 20

44) Bekanntestes Beispiel ist Jakob Fugger, der sich in Venedig die Fertigkeiten eines Großkaufmanns und Finanziers aneignete.

45) Die historische Herausbildung der oben skizzierten finanz- und wirtschaftspolitischen lnstrumente wurde zudem begleitet von der theoretischen Auseinandersetzung mit den politisch-ökonomischen Bedingungen des Warenverkehrs. Und in ihrer Entwicklung vom Bullionismus zum Fiskalismus, schließlich zur Theorie der Handelsbilanz und der Staatsschuld ist die Theorie des Merkantilismus allmählich dazu gelangt, die Funktionen des Staates für merkantile Akkumulation und manufakturmäßige Produktion systematisch zu erfassen. Vgl. hierzu: C. H. Wilson, Trade, Society and the State, in: The Cambridge Economic History, Bd. IV, a. a. O., S. 490 ff. und 575; Gustav Heinrich Hecht, Colbert's Politische und Volkswirtschaftliche Grundanschauungen, Freiburg 1898, S. 36. Gekenrm zeichnet ist diese Theorieentwicklung durch ihre Auseinandersetzung mit der nationalen Machtpolitik als einer Voraussetzung für die Durchsetzung ökonomischer Interessen. 
Autonomie der ersten bürgerlichen Konstitutionsprozesse vollziehen sich diese in einer vom Weltmarkt abhängigen Dynamik. Und die einzelnen nationalstaatlichen Entwicklungen sind nicht nur abhängig von der jeweiligen außenhandelspolitischen Machtposition, sondern auch von dem technologischen und wissenschaftlichen Fortschritt der merkantil führenden Nationen. Deshalb ist es nicht nur falsch, die Analyse der Entwicklung der kapitalistischen Produktion in England als generelles historisches Ablaufmodell für diesen Entwicklungsprozeß zu interpretieren, wie dies in mißverstandenem Anschluß an Marx vielfach geschehen ist; es ist ebenso verhängnisvoll, die Entwicklung der Staatsfunktionen und die besonders deutlich ausgeprägte souveräne Außenrepräsentanz, wie wir sie in England beobachten, als ein derartiges Muster zu begreifen. Denn was in England aus der Dynamik der nationalen ökonomischen Kräfte heraus zur Entstehung kam, das ist anderswo ganz bewußt den Briten abgeschaut worden, um international konkurrenzfähiger zu werden. Ebenso wie etwa Friedrich der Große das französische Steuersystem samt französischen Beamten in sein Land importierte, um bestimmte Entwicklungsrückstände aufzuholen, haben auch andere Herrscher eine bewußte Politik der Imitation ausländischer Errungenschaften verfolgt. Derartige Übernahmen lassen sich vielfach aufzeigen, und sie sind zumeist begleitet von einer lebhaften Diskussion über ihre politisch-ökonomische Brauchbarkeit. Analytisch sind sie zu werten als eine durch die internationale. Konkurrenzsituation hervorgerufene Beschleunigung der Entwicklung bestimmter Staatsfunktionen in den nachhinkenden Ländern.

Daß die strukturbestimmende Wirkung des Weltmarkts bereits in der Konstitutionsphase des bürgerlichen Staates sich entfaltet hat, gehört zu den in der Staatstheorie bislang vernachlässigten Aspekten. Soweit sich diese Feststellung auf den allgemeinen Basis-Überbau-Ansatz bezieht, ist festzuhalten, daß dieser fälschlicherweise vielfach tradiert wurde als Ansatz zur Analyse nationaler Systeme unter Absehung von deren internationalen Bestimmungen. Die staatliche Souveränität nach außen wird aber als politisches und juristisches Problem erst gesetzt durch die Konkurrenzbeziehungen, in deren Rahmen sie zu bestimmen ist.

Die Bedeutung der internationalen Konkurrenz für den konkreten nationalstaatlichen Konstitutionsprozeß läßt sich dokumentieren durch deskriptive Einzelanalysen. Systematisch ergibt sie sich im hier vorgetragenen Erklärungsansatz jedoch bereits aus dem konstitutiven Charakter der Außenfunktion. Denn diese kam zustande ja nur als die (macht-)staatliche Vertretung merkantiler Interessen, von Interessen also, welche sich entfalteten in einem ökonomischen Weltbild, das unter damaligen Produktionsbedingungen mit Recht davon ausging, daß des einen Gewinn unweigerlich des anderen Verlust bedeuten müsse. 


\section{Die Herstellung bürgerlicher Produktionsverhältnisse und die fomale Kon- stitution des bürgerlichen Staates}

Während der merkantilen Epoche erwächst aus der materiellen Konstitution des bürgerlichen Staates allmählich dessen formelle Konstitution als Rechtsstaat. Dieser Vorgang wird in der Regel beschrieben als die Durchsetzung bürgerlicher Freiheitsrechte gegenüber den fürstlichen Herrscherhäusern. Derartige Deskription ermangelt nicht nur des Erklärungswertes für den hier angesprochenen Prozeß, sie ist zudem - und dies scheint mir ein bislang in der wissenschaftlichen Diskussion weitgehend vernachlässigter Gesichtspunkt zu sein - in relevanter Weise unvollständig. Denn in der merkantilen Übergangsphase vollzieht sich zwar einerseits die Entwicklung zur Durchsetzung der abstrakten Herrschaftsformen des Rechtsstaates, zum anderen jedoch erfüllt die Staatsgewalt zusätzlich solche Funktionen für die sich herausbildende bürgerliche Gesellschaft, die zwar in der Rechtsform von Gesetzen auftreten, ihrem Inhalt nach aber offene Gewalt und eindeutige soziale Standeszuweisungen beinhalten, wie sie unvereinbar sind mit der bürgerlichen Rechtsauffassung. Dieser Widerspruch ist historisch bedeutsam: er markiert den in den verschiedenen Ländern je nach Stand der Produktionsverhältnisse und zu unterschiedlichen Zeiten stattfindenden Einschnitt zwischen der materiellen und der verfassungsmäßigen Durchsetzung des bürgerlichen Rechtsstaates.

Diesen Widerspruch im Konstitutionsprozeß des bürgerlichen Staates will ich versuchen, in kurzen Zügen abschließend zu kennzeichnen.

Behandeln wir zunächst denjenigen Aspekt, welcher die Grundlage bildet der bürgerlichen Theorie des Rechtsstaates und der Form bürgerlicher Herrschaft. Auf einer sehr allgemeinen Ebene kan er begriffen werden als die institutionelle Sicherung einer staatlichen Garantie für Verfahrensweisen des Warenaustauschs. Derartige Verfahrensweisen haben sich nicht erst entwickelt in der Phase des Spätfeudalismus, sie sind in bestimmtem Maße Grundlage jedes funktionierenden Warenverkehrs. Dieser Zusammenhang erklärt auch, weshalb zur Herausbildung des modernen bürgerlichen Rechts Kategorien des antiken römischen Rechts formal übernommen werden konnten. Waren die im Vertragsrecht gipfelnden Regelungen des Äquivalententausches in der spätrömischen Gesellschaft noch beschränkt auf bestimmte Teilbereiche, so erforderte die Ausdehnung des Warentausches in der Phase des Merkantilismus die Ausdehnung der Regelungen des Tausches. Dies läßt sich vereinfacht sehr anschaulich erklären: In einer Gesellschaft, die vorwiegend noch für den eigenen Bedarf produziert, findet der Warenverkehr entweder statt als direkterTauschhandel - und in diesem Fall ist die Befriedigung der gegenseitigen Interessen weitgehend als gesichert anzusehen, sofern nicht einer der Tauschpartner betrügerisch handelt. Bei örtlich begrenztem Warenverkehr tritt in solchem Fall soziale Sanktion mit ihren ökonomischen Folgen auf. Sofern Geldgeschäfte getätigt und vor allem Kredite eingeräumt werden, geschieht dies in der Regel aufgrund persönlicher Bekanntschaft oder Empfehlung. Dieses Verfahren finden wir lange Zeit noch praktiziert im Kolonialhandel. Es impliziert ein mehr oder minder persönliches Vertrauensverhältnis zwischen sich gegenseitig kreditierenden Handelshäusern. Um die derart persönliche Beschränkung des Warenverkehrs aufzuheben und damit die Auswei- 
tung des nationalen und des internationalen Handels überhaupt erst zu ermöglichen, bedarf es einer übergeordneten Garantie der gegenseitigen Abmachungen (46). Diese Garantie hat im Zeitalter des Merkantilismus zunehmend die Staatsmacht übernommen, d. h. sie stand zur Verfügung zur Durchsetzung freiwillig abgeschlossener Verträge mit Rechtsgewalt. Derartige Rechtsgarantie mittels Staatsgewalt galt zum einen innerhalb einer Nation, zum anderen aber auch in bestimmten internationalen Beziehungen. Damit nicht ein Individuum durch unlautere Handlungsweisen den Interessen der Gesamtheit einer nationalen Handelsbourgeoisie schaden konnte, übernahm der Staat auch insoweit Rechtsgarantie, als er ausländischen Vertragspartnern ermöglichte, ihn zur Durchsetzung von Rechtsansprüchen gegen einheimische Bürger anzurufen. Umgekehrt bot die Staatsgewalt Hilfe an zur Eintreibung von Ansprïchen im Ausland. Die Durchführung derartiger internationaler Garantieübernahmen, gleichzeitig aber die Notwendigkeit, die ständig steigenden Kosten für Handelskriege zu minimieren, erforderten die Herausbildung von Vertragsbeziehungen auch zwischen Nationalstaaten: Sie förderten die Entwicklung des internationalen Rechts.

Diese Entwicklung erfolgte im Widerspruch zur Theorie des souveränen Staaies, welche mit ihrem Begriff von der Zuhöchstheit des einen Staates die Anerkennung von anderen Staaten ausschloß (47). Aber gerade aus der in diesem Sinne konsequenten nationalen Politik, dem Streben nach merkantiler Weltherrschaft hat sich real die Notwendigkeit der Einhaltung bestimmter Verträge zur Sicherung des bereits eroberten Handelsimperiums herausgestellt, und theoretisch resultiert aus diesem Zusammenhang die Entwicklung des internationalen Rechts (48). Dieses entsteht in der Phase der relativen Konsolidienung der großen Kolonialreiche und entwickelt sich theoretisch als Kritik an der Souveränitätstheorie, die ihrerseits den adäquaten Ausdruck bildet der ersten kolonialen Eroberungen. Der merkantile Widerspruch zwischen Souveränität und internationaler Gemeinschaft, der historisch aus dem 17. und 18. Jahrhundert stammt, ist ein bis heute fortdauerndes Merkmal nationalstaatlicher Politik.

Auch im Innern vollzieht sich die Konstitution des bürgerlichen Staates in realer und theoretischer Auseinandersetzung mit der fürstlichen Souveränität. Der zentrale Inhalt der Entwicklung zum bürgerlichen Rechtsstaat liegt in der $A b s t r a k$ tion der Staatsgewalt von den gesellschaftlichen Beziehungen. Der Rechtsstaat greift nicht unmittelbar in diese ein, sondern er fungiert als Garant ihrer Existenz. Durchgesetzt werden mußte eine derartige Bestimmung der Staatsgewalt gegen die frühe-

46) Im übrigen auch einer Wandlung des Fremdenrechts; die Entwicklung einer nicht nur auf einzelne Personen bzw. Handelsgesellschaften bezogene fremdenfreundliche Politik und bestimmter Schutzgarantien gehört - trotz feststellbarer Ausnahmen - mit zu den charakteristischen Merkmalen der merkantilen Epoche.

47) Einführend hierzu: Hans Kelsen, Der Wandel des Souveränitätsbegriffs, (erstmals 1931), wieder abgedruckt in: Volkssouveränität und Staatssouveränität. Hrsg. Hanns Kurz, Darmstadt 1970, S. $166 \mathrm{ff}$.

48) So auch Gustav Heinrich Hecht, Colbert's Politische und Volkswir tschaftliche Grundanschauung, a. a. O., S. 34: „Indem der Merkantilismus im Prinzip jedes Völkerrecht negiert, führt er gerade durch diese Negierung zur Begründung der Ideen, auf denen sich das moderne europäische Völkerrecht aufbaut." 
ren einseitigen Verträge der Fürsten mit ihren Untertanen (z, B. mit den Städten) (49), gegen die explizite standesmäßige Zuweisung von sozialen Stellungen sowie gegen staatlich institutionalisierte Störungen des freien Warenverkehrs. In der revolutionären Theorie des Bürgertums wurden alle diese Ziele zusammengefaßt in der Parole: ,Kampf der fürstlichen Willkür'.

Und doch wurde zur selben Zeit, als derart revolutionär die Bürgerfreiheit erkämpft wurde, staatliche Gewalt unmittelbar eingesetzt zur Herstellung ganzbestimmter gesellschaftlicher Beziehungen. In diesen Zusammenhang fallen vor allem die Kriminalisienung der Armut und die ihr entsprechende merkantile Arbeitsgesetzgebung. Systematisch ist diese Entwicklung zu fassen als die gewaltsame Herstellung von Lohnarbeitsverhältnissen; der Form nach handelt es sich um eine Ablösung der früheren Fürsten- und Christenpflicht zur Mildtätigkeit. Diese war resultiert sowohl aus der Gesellschaftsauffassung des Mittelalters, deren soziale Wirksamkeit abhing von der tatsächlichen Errüllung caritativer Pflichten durch den Herrn, als auch aus der Notwendigkeit, soziale Störungsfaktoren zu minimieren. Im Rahmen noch weitgehend feudaler Sozialbeziehungen war Armut weniger ein individuelles Schicksal, als vielmehr eine gemeinsame Aufgabe. Was unter anderem auch darin zum Ausdruck kam, daß feudale Abgaben oft für eine Gemeinde als ganzes und nicht für die einzelne Familie festgesetzt wurden. Während der merkantilen Ubergangszeit wurde Armut nicht nur zum individuellen Schicksal, sondern sie wurde sozial tendenziell transformiert zu persönlicher Schuld. So finden wir überall in den europäischen Staaten eine wachsende Zahl von Arbeits- und Zuchthäusern, Armenhäusern und Schuldtürmen, deren Belegschaften zunehmend vermietet wurden an private Manufakturproduzenten bzw. eingesetzt wurden zur staatlichen oder fürsteneigenen Manufakturproduktion (50). In einer der wichtigsten theoretischen Arbeiten der Merkantilphase, dem ,Treatise of Taxes and Contributions" hat 1662 William Petty dieser Entwicklung und zugleich der klassischen Arbeitswertlehre ihr Programm geschrieben, indem er vorschlug, jede Strafe umzuwandeln in Zwangsarbeit, weil dies zusammen mit dem verfügbaren Arbeitsvolumen den nationalen Reichtum vergrößern würde. Die Argumentation zugunsten der neuen Weise, Reichtum zu schaffen, wird besonders deutlich im folgenden Zitat aus Petty's Schrift:

49) Dieser historische Zusammenhang ist zu berücksichtigen bei der Analyse frühbürgerlicher Vertragstheorien.

50) Vgl. hierzu für einzelne Länder: Friedrich Lütge, Deutsche Sozial- und Wirtschaftsgeschichte. 2. Aufl. Berlin-Göttingen-Heidelberg 1960, S. 384 f.; Franz Erle, Beiträge zur Geschichte und Reform der Armenpflege. 1881; Gustav Heinrich Hecht, Colbert's Politische und Volkswirtschafthche Grundanschauung, a. a. O., S. 66 f.; C. H. Wilson, Trade, Society and the State, a. a. O., S. $542 \mathrm{ff}$.; Sidney and Beatrice Webb, English Local Government: English Poor Law History. London-New York-Toronto-Bombay-CalcuttaMadras 1927; vgl. hierzu auch Colbert's Galeerenpolitik bei Eugene L. Asher, The Resistance to the Maritime Classes, a. a. O., S. 7 
,Warum sollten nicht zahlungsunfähige Diebe lieber mit der Sklaverei bestraft werden als mit dem Tod? Denn als Sklaven können sie fast ohne alle Unkosten zu so viel Arbeit gezwungen werden, als ihre Konstitution zuläßs $t$. Auf diese Weise werden dem Cornmonwealth zwei Arbeitskräfte gewonnen, während anders ihm eine entzogen worden wäre. "(51)

Handelt es sich in England und Frankreich vor allem um die Kriminalisierung von Armut, so ist in den preußischen Provinzen die Manufakturperiode teilweise eingefuihrt worden unter Beibehaltung feudaler Bindungen. Die gelegten Bauern hatten zwar ihr Land und ihre Produktionswerkzeuge eingebüßst, die Freiheit zur Lohnarbeit dabei jedoch nicht zugleich gewonnen. Ein Großteil der Manufakturbetriebe ist deshalb bis ins 19. Jahrhundert hinein in fürstlichem Besitz (52), und die neue Produktionsform wurde durchgesetzt unter Beibehaltung der unmittelbaren feudalen Herrschaftsbeziehungen.

Gewaltbeziehungen jedenfalls kennzeichnen allgemein die Entwicklung in Europa, wenn Waisenhäuser an Produzenten vermietet wurden und jeder ins Arbeitshaus kam, der nicht schlüssig beweisen konnte, kein Vagabund zu sein (53). Für Großbritannien ist nachgewiesen, daß, solange britische Handelskompanien ihre Interessen in den Festlandskolonien Amerikas noch durchzusetzen vermochten, die Zahl der Festsetzungen in Schuldtürmen mit anschließender Deportation in die Kolonien sich häuften (54). Derartige Deportierte hatten im 17. Jahrhundert in den amerikanischen Festlandskolonien zunächst dieselbe soziale und rechtliche Position, wie die aus Afrika importierten Sklaven. Zusammen mit ihren Leidensgenossen in Europa bildeten sie das Arbeitspotential für die neuen Produktionsformen, das anders nicht $\mathrm{zu}$ gewinnen gewesen wäre. $\mathrm{Zu}$ derart unmittelbaren Maßnahmen gesell ten sich indirekte. So die zeitweilige Heraufsetzung des Getreidepreises, um auch Frauen und Kinder in den Arbeitsprozeß zu zwingen, ind schließlich Einzelbestimmungen der Arbeitsgesetzgebung, die, wie etwa die berïhmten Tudor-Statute, die Lehrzeit auf sieben Jahre festsetzen und sie bestimmten als ein Bindungsverhältnis, welches den Gebundenen nahezu sämtlicher Personenfreiheiten entblößte. Derartige Gesetzgebungen werden in der Regel beurteilt als Ausläufer der Feudalzeit, was einerseits übersieht, daß die berühmten Theoretiker der sog. klassischen Theorie der bürgerlichen Ökonomie bis hin zu Mill und Bentham derartige Gesetzgebungen ebenfalls propagierten (55), was aber vor allem uber die materielle Wirkung solcher

51) The Economic Writings of Sir William Petty. Bd. I, Cambridge 1899, Bd, 1, S. 68 (Ubersetzung nach Werner Hofmann)

52) Vgl. C. H. Wilson, Trade, Society and the State, a. a. O., S. $555 \mathrm{ff}$.

53) E. P. Thompson, The Making of the English Working Class. 1963; Wille, English Poor Law History I, S. 102-114; Lettres de Colbert, Bd. III, S. 1 ff. et passim; C. J. RibtonTurner, History of Vagrants and Vagrancy. London 1887.

54) Herrick A. Cheesman, White Servitude in Pennsylvania. Philadelphia 1926, S. 266; Lewis Cecil Gray, History of Agriculture in the Southern United States 1607-1860. Washington D. C. 1916, S. 343; Lawrence Henry Gipson, The British Empire before the American Revolution. 13 Bde., New York 1936-1967. Bd. II, S. 95 ff., Bd. III, S. 192 ff.; Richard B. Morris, Government and Labor in Early America, New York 1946, S. 337 ff.

55) Vgl. hierzu allgemein Lionel Robbins: The Theory of Economic Policy in English Classical Political Economy. London 1952, S. 95-100; Eli R. Heckscher, Mercantilism. Bd. II, revised Ed. London 1962 (erstmals 1931), Kapitel V. 
Legislation und Jurisdiktion sich hinwegsetzt. Denn diese staatlichen Maßnahmen dienten dazu, die fortschrittliche kapitalistische Produktionsweise und die Entwicklung der Kolonien gesellschaftlich durchzusetzen. Es handelt sich (zu großen Teilen) urh eine Legislation und Jurisdiktion zur Herstellung von lohnarbeitsähnlichen Verhältnissen, und wer die Anwendung unmittelbarer Herrschaftsgewalt für die Sicherstellung der Produktion in den sich entwickelnden europäischen Nationen einmal zu studieren beginnt, der hält die Anwendung der Sklaverei in den europäischen Kolonien nicht mehr für jene extraordinäre Erscheinung, als die sie von den Philantropen späterer Jahre gebrandmarkt wurde.

Was bei den angesprochenen Maßnahmen in Mitteleuropa noch feudal anmutete, ist der unmittelbare Eingriff der Staatsgewalt in die gesellschaftlichen Verhältnisse, die Beteiligung an deren Herstellung im Unterschied zur rechtsstaatlichen Garantie ihrer Existenz. Zum Teil drückt sich dies auch aus in der Art, wie die Gesetze zeitgenössisch interpretiert wurden, nämlich als Zuchtinstrumente des Fürsten als Landesherren, als Ausfluß seiner persönlichen Herrschaft (56). Die absolutistische Form fürstlicher Souveränität wurde fungibel gemacht für die Durchsetzung bürgerlicher Produktionswerhältnisse.

Für die Theorie des bürgerlichen Staates als Rechtsstaat jedenfalls scheint es mir von eminenter Bedeutung zu sein, daß während der Merkantilzeit der bürgerliche Staat sich in seinen historisch grundlegenden ökonomischen Funktionen und Organisationsformen bereits herausbildet, daß aber die für ihn typische rechtsstaatliche Entwicklung sich noch beschränkt auf die Zirkulationssphäre, welcher sie materiell entstammt. Die Ausdehnung des Prinzips vom Aquivalententausch auch auf die Produktionssphäre, die Einbeziehung des Arbeitsvertrages in den freien Warenverkehr, sie wird erst möglich nach der weitgehend gewaltsamen Herstellung von Lohnarbeitsverhältnissen. Erst jetzt auch ist die für den Rechtsstaat typische Abstraktion von den bürgerlichen Geschäften möglich, erst jetzt gilt die formale Gleichheit des Bürgers vor dem Gesetz. Staatliche Gewalt erhält fortan subsidiären Charakter, wird - in Jurisdiktion, in Polizei- und Militäreinsatz - ausgeübt zur Sicherung der etablierten gesellschaftlichen Verhältnisse. Diese rech tsstaatliche Abstraktion aber markiert historisch den Übergang von der materiellen Konstitution des bürgerlichen Staates zu seiner verfassungsmäßigen. Von jetzt an begründet sich die Notwendigkeit des bürgerlichen Staates nicht mehr durch die spezifischen Beschränkungen der Einzelkapitale bzw. durch deren in der Konkurrenz verdeckten oder bedrohten gemeinsamen Interessen, sondern vor allem dadurch, daß iber die scheinbare Freiheit der Arbeitsvertragsbeziehungen und über die scheinbare Gleichheit der Revenuequellen in der bürgerlichen Gesellschaft die scheinbare Neutralität

56) In diesem Zusammenhang ist z. B. zu erinnern an das, Stockregiment ${ }^{5}$ Friedrich Wilhelm I. Vgl. Friedrich Lütge, Deutsche Sozial und Wirtschaftsgeschichte, a. a. O., S. 385 
des bürgerlichen Staates gesetzt wird (57). Die unmittelbar ökonomischen Funktionen des bürgerlichen Staates werden um solche der Legitimation erweitert, und diese manifestieren sich zunächst und in erster Linie in den spezifischen Formen bürgerlicher Staatlichkeit.

57) Die von Sybille von Flatow und Freerk Huisken vorgenommene systematische Ableitung des bürgerlichen Staates (vgl. ProKla Nr. 7, Mai 1973), die von der Oberflächenstruktur des (entfalteten) Kapitalverhältnisses ausgeht, muls also notwendigerweise die Legitimationsfunktion des bürgerlichen Staates zur zentralen erheben. Demgegenüber stellt sich die Frage, ob nicht die hier entwickelte historische Dominanz ökonomischer Staatsfunktionen auch systematischen Charakter hat. Aus der gesellschaftlichen Oberflächenstruktur des Kapitalverhältnisses wäre dann die spezifische Form der Besonderung des bürgerlichen Staates abzuleiten, während diese Besonderung selbst aus der Notwendigkeit der Erstellung allgemeiner Produk tionsbedingungen folgen würde. 